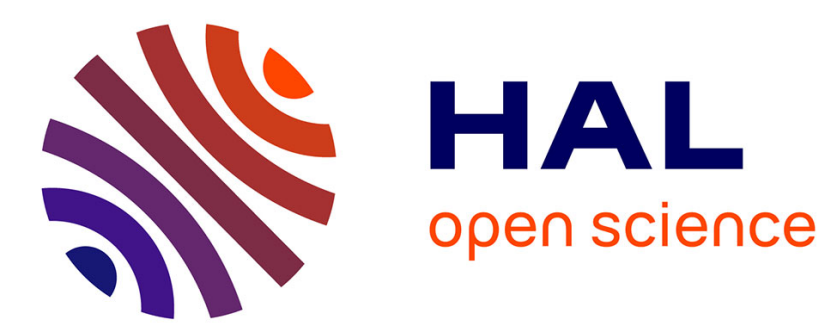

\title{
Scénariser les possibles énergétiques. Les gaz de schiste dans la matrice des futurs
}

Francis Chateauraynaud, Josquin Debaz

\section{To cite this version:}

Francis Chateauraynaud, Josquin Debaz. Scénariser les possibles énergétiques. Les gaz de schiste dans la matrice des futurs. 2012. hal-00766121

\section{HAL Id: hal-00766121 \\ https://hal.science/hal-00766121}

Preprint submitted on 17 Dec 2012

HAL is a multi-disciplinary open access archive for the deposit and dissemination of scientific research documents, whether they are published or not. The documents may come from teaching and research institutions in France or abroad, or from public or private research centers.
L'archive ouverte pluridisciplinaire HAL, est destinée au dépôt et à la diffusion de documents scientifiques de niveau recherche, publiés ou non, émanant des établissements d'enseignement et de recherche français ou étrangers, des laboratoires publics ou privés. 


\section{Scénariser les possibles énergétiques. Les gaz de schiste dans la matrice des futurs.}

Texte à paraître dans la revue Mouvements en 2013 - version du 17 décembre 2012

Francis Chateauraynaud et Josquin Debaz (GSPR-EHESS)

La scapulomancie désigne un vieil art divinatoire consistant à interpréter les craquelures d'un os plat soumis à une forte chaleur. Dans le dossier des gaz de schiste, il est aussi question de provoquer et d'interpréter le résultat de fractures, longues de quelques dizaines à quelques centaines de mètres dans le sous-sol. Ces opérations mécaniques permettent de récupérer du gaz naturel dans ce qu'il est convenu d'appeler la roche-mère. Avant toute exploitation, les industriels effectuent des forages exploratoires et doivent également interpréter correctement la fracture pour estimer les futurs potentiels du site.

En France, l'envergure et la rapidité de la mobilisation contre l'utilisation de la fracturation hydraulique (fracking ou bydraulic fracturing dans les mondes anglophones) ont frappé tous les commentateurs. Avant novembre 2010, peu d'acteurs étaient concernés par cette technique dont l'utilisation industrielle a pourtant débuté dans les années 1980 aux États-Unis, avec les premiers forages horizontaux de la firme Halliburton. L'intensité de la mobilisation peut s'expliquer par la variété des enjeux portés par le dossier : risques de pollution de l'eau, atteinte de milieux jusqu'ici préservés (parfois liés à des luttes historiques de défense d’un territoire), confrontation de systèmes juridiques anachroniques (droit de l'environnement récent et droit minier ancien). L'appropriation citoyenne des problématiques environnementales, amplifiées par le Grenelle de l'environnement, ainsi que l'existence de débats et de luttes en Amérique du Nord, a aussi pesé dans l'organisation de la protestation au niveau national'. La vitesse de propagation de la « controverse » procède également de la conjonction de trois processus critiques :

- Le développement d'un espace de calcul dans lequel politiques énergétiques et environnementales font l'objet d'évaluations croisées (sur fond d'expression politique de l'alerte climatique dont les concepts et les outils résultent de quarante années de sommets de la Terre) ;

- le dévoilement de permis exclusifs de recherche accordés depuis plusieurs années et la réactivité des milieux écologiques décidés à ne pas se laisser prendre de vitesse par les industriels ;

\footnotetext{
${ }^{1}$ Francis Chateauraynaud et Josquin Debaz avec la collaboration de Pierrick Cézanne-Bert, «L'affaire des gaz de schiste. Anatomie d'une mobilisation fulgurante», Socio-informatique et argumentation, 19 décembre 2011. http://socioargu.hypotheses.org/3262.
} 
- enfin, l'idée que le lancement de programmes d'exploitation d'hydrocarbures nonconventionnels présenterait un caractère irréversible, en termes environnemental et industriel.

Dans cet article, nous abordons l'analyse du conflit des gaz de schiste sous l'angle des scénarisations du futur. Les questions énergétiques sont depuis fort longtemps au cœur de modèles économiques qui alimentent tout à la fois des prospectives et des prévisions, mais aussi des promesses et des prophéties. Les formes de protestation et les frictions révélées par ce dossier font apparaitre des visions du futur contrastées, issues d'une production continue de conjectures autour des enjeux énergétiques. Les différents acteurs partagent-ils un même espace de calcul et de raisonnement sur la question de la finitude des ressources (pétrolières en particulier) ? Nous pouvons partir de l'hypothèse, aujourd'hui largement partagée, selon laquelle le surgissement des gaz de schiste a contribué à une réactualisation des multiples scénarios énergétiques. L'Agence Internationale de l'Energie (IEA), écrit dans le préambule d'un rapport récent :

La carte énergétique mondiale est en pleine évolution, ce qui peut avoir de profondes répercussions sur les marchés et les échanges d'énergie. Transformée par la résurgence de la production pétrolière et gazière aux États-Unis, elle pourrait se trouver remodelée également par l'abandon du nucléaire dans certains pays, par la croissance rapide et continue de l'utilisation des technologies solaires et éoliennes et par la généralisation de la production de gaz non conventionnel au niveau mondial. ${ }^{2}$

Si l'on prend la peine de regarder les énoncés concernant les futurs énergétiques, on relève des tensions épistémiques et cognitives entre plusieurs modalités d'argumentation: il s'agit de caractériser un processus en cours, encore ouvert, de pointer des conséquences plausibles mais encore incertaines (comme dans «ce qui peut avoir de profondes répercussions»...) et d'organiser la liste des paramètres ou des facteurs à prendre en compte dans l'évaluation des «mix énergétiques», du report dans le futur d'un peak oil définitif, ou encore des chances différentielles de garantir une indépendance énergétique. Si la plupart des acteurs tentent de fixer des scénarios sur la base de modèles de transformation réalistes, on observe de multiples variations dans la manière d'agencer la factualité, l'interprétation des signes et des tendances, et l'orientation normative vers des décisions politiques et économiques, généralement qualifiées d'urgentes.

\section{Une sociologie des futurs}

Plusieurs enquêtes récentes ont mis en évidence l'importance d'une sociologie pragmatique des futurs capable d'éviter le double écueil de l'heuristique de la peur (Jonas), reformulée par JeanPierre Dupuy sous la forme du catastrophisme éclairể, et du relativisme qui consiste à rapporter toute construction du futur à ses conditions de production locale - en renouant avec la vieille

\footnotetext{
2 International Energy Agency, World Energy Outlook 2012, 12 novembre 2012.

${ }^{3}$ Jean-Pierre Dupuy, Pour un catastrophisme éclairé, Paris, Seuil, 2002.
} 
conception augustinienne des trois présents ${ }^{4}$. On a pu montrer en confrontant les visions du futur à l'œuvre à propos du nucléaire, du climat, des OGM ou des nanotechnologies, que les différentes constructions du futur n'avaient pas les mêmes portées dans les joutes argumentatives ${ }^{5}$. Une vision du futur a d'autant plus de chances de s'imposer qu'elle parvient à lier de façon cohérente une échelle temporelle, un modèle de transformation et une logique d'action ${ }^{6}$. A partir de ce jeu de contraintes, plusieurs régimes d'énonciation du futur sont disponibles. La scénarisation, telle que l'entendent les acteurs, va ainsi jouer à la fois de la veille et de l'anticipation, de la prospective et de la prévision, en tirant parfois vers la promesse ou la prophétie. En composant leur régime d'énonciation, les acteurs entreprennent de "paramétrer» leur espace de calcul, un bon scénario servant alors aux institutions comme aux personnes et aux groupes pour organiser leurs actions, à court et long terme ${ }^{7}$. Il s'agit de fixer une représentation pour une certaine durée et de faire en sorte qu'elle obtienne un certain crédit public de manière à produire les effets performatifs attendus. Dans cette opération qui doit être régulièrement réitérée, même dans le cas de prévisions officielles sur des phénomènes globaux (démographie, croissance, climat, gisements et ressources naturelles...), le degré de pertinence des visions du futur dépend de l'angle de vision adopté par leurs porteurs : certains acteurs auront ainsi tendance à fermer l'angle et à viser une direction déterminée là ou d'autres choisiront l'ouverture maximale de façon à prendre en compte des scénarios alternatifs, par exemple en faisant l'hypothèse de la montée d'innovations, de marchés ou de mobilisations encore à l'état embryonnaire.

\section{Les gaz de schiste font-ils bouger les échelles temporelles?}

Du fait d'un coût d'extraction élevé, l'investissement dans les gaz de schiste s'accompagne d'un changement de stratégie de la part des pétroliers dont l'espace de calcul est marqué par une économie de basculements. L'engagement précoce sur une nouvelle ressource permet de s'y lancer avant les autres et de profiter des aubaines d'un marché avant sa mise en tension. Mais cette stratégie demande de confronter les données d'investissement au risque d'une obsolescence rapide des techniques face au développement de la recherche dans le domaine. De même se lancer dans une série de concentrations d'entreprises pour palier à un investissement tardif ou pour s'imposer par la taille sur un marché agressif, implique une robustesse économique suffisante face aux aléas des cours des différents hydrocarbures. Ainsi le gaz non conventionnel est intéressant au plan financier tant que son cours et celui du brut restent élevés, mais si un événement comme une baisse de la demande en pétrole ou une production importante de gaz (liée précisément à l'élan vers les gaz de schiste) perturbe les marchés financiers, les risques d'effet de massue ou de trou de trésorerie sont importants. Outre ces calculs financiers, le coût

\footnotetext{
${ }_{4}^{4}$ «On ne peut dire, à proprement parler, qu'il y ait trois temps, le passé, le présent et le futur ; mais peut-être serait-il plus juste de dire : Il y a trois temps, le présent des choses passées, le présent des choses présentes, le présent des choses futures. » Saint Augustin, Les confessions, Livre XI, ch. XX. 26.

${ }^{5}$ Voir F. Chateauraynaud, «Des prises sur le futur. Regard analytique sur l'activité visionnaire », in D. Bourg et alii, $D u$ risque à la menace. Retour sur la société du risque, à paraître en mars 2013. Version pre-print disponible en ligne, http://www.gspr-ehess.com/documents/articles/FC-Prises-sur-le-Futur-janv2012.pdf.

${ }^{6}$ C'est bien souvent parce qu'ils ne parviennent pas à articuler de façon cohérente ces jeux de contraintes que les « experts », "prévisionnistes » ou «prospectivistes », donnent prise à la critique de l'arbitraire des croyances imposées à coup d'arguments d'autorité. Voir Nassim Nicholas Taleb, Le cygne noir : La puissance de l'imprévisible, Paris, Les Belles Lettres, 2008.

${ }^{7}$ Voir par exemple le scénario énergétique de l'ADEME produit avant le débat sur l'énergie de 2012. «L'Ademe propose son scénario énergétique d'ici 2050 », Actu-Environnement, 13 novembre 2012.
} 
économique de l'opération doit incorporer une éventuelle marge d'erreur dans l'évaluation des capacités d'un gisement, sans oublier les réparations des dommages environnementaux potentiels - lesquels dépendent également de l'évolution de la réglementation.

Pour les groupes mobilisés au nom des populations locales, l'enjeu est de reprendre en main l'avenir de leur territoire, en contestant les projets portés par les multinationales pétrolières soutenues par l'État. Quand l'économie d'une région repose sur la préservation de son environnement, ses porte-parole exigent que leur point de vue soit pris en compte dans les équations financières aux niveaux national et international. Ce faisant, ils doivent outiller leur cause en s'emparant des éléments de calculs économiques et rétroagir sur les paramètres les plus pertinents. Le travail d'analyse critique doit alors maîtriser les échelles temporelles qui se croisent dans le dossier. Dans le modèle matriciel du futur, la catégorie du «court terme » engage un « futur qui est déjà là ». On comprend mieux à cet égard l'urgence de l'action, puisque des permis de recherche ont été négociés par les industriels dès janvier 2006 (ce qui serait attesté par le " permis de Foix » exhumé par l'enquête des opposants). Dans la même période, le débat est déjà vif au Canada tant autour des conditions environnementales, les opposants s'appuyant sur le mauvais exemple de la Pennsylvanie, qu'autour des montages financiers et fiscaux qui relient les industriels et le gouvernement de Stephen Harper. L'opposition aux gaz de schiste est ainsi apparue comme la contestation de manœuvres tirant profit de la vétusté du droit minier pour contourner l'épreuve de consultation des populations qui s'impose sur tous les dossiers touchant l'environnement.

A moyen terme, l'enjeu est plutôt la mise en balance d'un statu quo économique pendant encore quelques années et d'un basculement immédiat dans une économie post-pétrole. A long terme, le problème des acteurs est de savoir si l'injection de produits chimiques, qui, selon de multiples hydrogéologues ou géochimistes, remonteront tôt ou tard vers des strates plus élevées, vaut les impacts qu'elle produira. La contestation du double calcul, coût-bénéfice d'un côté et bénéficerisque de l'autre, met en évidence l'asymétrie entre ceux qui vont profiter de l'opération et ceux qui ont de fortes chances d'en supporter les conséquences négatives, réelles ou potentielles (ce qui est modélisé par les économistes dans le jeu de langage des « externalités »). Outre l'asymétrie sociale ou spatiale engendrée par ce processus, une asymétrie temporelle se fait jour: forts des exemples marquants - comme l'amiante ou les pollutions chimiques persistantes - le report sur les générations futures est jugé inacceptable, ce qui caractérise la puissance d'expression propre aux topiques environnementales, qui portent à raisonner à l'échelle du siècle, ou au-delà, comme pour le climat, la biodiversité ou la gestion des déchets radioactifs. Autrement dit, le long terme fournit une matrice critique des futurs qui entre en conflit avec la logique du court ou du moyen terme dans laquelle raisonnent les acteurs économiques.

\section{Une lecture argumentative par les marqueurs de rupture}

$\mathrm{Au}$ plan analytique, l'attention portée aux marqueurs de rupture ou d'irréversibilité tels que «désormais » permet de mettre à jour les constructions temporelles des acteurs ${ }^{8}$. Prenons par

\footnotetext{
8 Francis Chateauraynaud et Marianne Doury, " "Désormais..." Essai sur les fonctions argumentatives d'un marqueur de rupture temporelle ", Argumentation et Analyse du Discours, 4, 2010, mis en ligne le 15 avril 2010, Consulté le 16 octobre 2012. URL : http://aad.revues.org/772.
} 
exemple le raisonnement qui pose comme inéluctable la baisse des réserves de pétrole, avec pour conséquence l'augmentation du prix du pétrole. Son porteur va logiquement se saisir des atouts du gaz comme substitut financièrement compétitif. Dans la séquence suivante, l'usage de « désormais » impose l'idée d'un basculement irréversible : les prix du gaz aux États-Unis seront toujours plus compétitifs qu'en Europe à moins que les gaz de schiste y soient également exploités.

Pourra-t-on longtemps se plaindre de la flambée des prix de l'énergie et refuser l'exploitation des gaz. de schiste au nom des menaces qu'ils font peser sur l'environnement (fracturation bydraulique, pollution des nappes phréatiques...)? Le pire n'est jamais certain. L'État a les moyens, techniques et juridiques, d'en encadrer strictement la production. [...] Les gaz de schiste dessinent un scénario économique moins sombre que certaines prédictions écologistes : un renforcement de l'indépendance énergétique [...] Aux États-Unis, la production à grande échelle des shale gas depuis 2009, où ils représentent aujourd'bui $23 \%$ de la consommation, a commencé à modifier la donne. Le prix du gaz a été divisé par cinq depuis son sommet de l'été 2008. Désormais trois fois moins cher que sur le Vieux Continent, il donne un avantage compétitif indéniable à certains secteurs de l'industrie américaine (chimie, pétrochimie...). Tout indique que les prix du pétrole continueront d'angmenter dans les prochaines années, à mesure que les réserves déclineront et que la demande des pays émergents augmentera. C'est donc tout le "mix énergétique » qu'il faut repenser - sans faiblesse visà-vis de la défense de l'environnement, mais sans tabou. ${ }^{9}$

Ce raisonnement repose sur un modèle de transformation linéaire. Mais le renversement annoncé, en termes d'avantage économique, d'un hydrocarbure sur un autre n'entraine pas pour autant un accord unanime quant aux priorités qui en découlent. Chez Arnaud Gossement, juriste de l'environnement, l'injonction économique immédiate ne doit pas prévaloir sur un calcul à plus long terme impliquant à la fois des risques irrémédiables et des effets pervers sur des alternatives énergétiques. Ce qui le conduit à invoquer une autre figure d'irréversibilité, celle de l'évolution du droit de l'environnement rendant nécessaire la participation du public à la prise de décision :

Au fil des années, l'évaluation environnementale et la participation du public ont cependant pris une part plus large dans les procédures définies par le droit minier comme le présent rapport le démontre. Cette évolution est largement le résultat du progrès du droit international et européen de l'environnement auquel le droit minier ne pouvait se soustraire. Désormais, un choix doit être opéré, non pas entre production et protection, mais sur l'endroit précis où placer le curseur. S'il est inconcevable de s'engager, pour des raisons financières de court terme, dans la voie de productions industrielles susceptibles de comporter des risques graves et irréversibles de pollution, il n'en demeure pas moins que l'interdiction générale et absolue ne saurait constituer le seul instrument de régulation de l'accès au sous-sol au risque de mettre également en péril des activités économiques dont le développement est encouragé par la collectivité, comme les énergies renouvelables. ${ }^{10}$

\footnotetext{
${ }^{9}$ « Le pétrole flambe, le gaz de schiste attend », Le Monde, 29 février 2012.

10 Arnaud Gossement, Droit minier et droit de l'environnement - Eléments de réflexion pour une réforme relative à l'évaluation environnementale, à l'information et à la participation du public, rapport au Ministère de l'écologie, du développement durable, des transports et du logement, 12 octobre 2011, 24-25.
} 
Comme dans la plupart des dossiers, les écologistes mettent au premier plan le calcul à long terme, seul à même de répondre à la problématique globale des changements environnementaux. Pour Greenpeace, la controverse n'a que trop duré et l'heure impose de définir une stratégie énergétique alternative :

Après des années de controverses sur les politiques à adopter pour lutter contre les changements climatiques et le développement des infrastructures énergétiques au Canada, il est désormais reconnu et accepté que le pays a besoin d'une stratégie énergétique animée par une vision d'avenir [...] Quelle que soit l'orientation adoptée, il est primordial que le choix du Canada se fasse sur la base d'un débat public éclairé et non derrière des portes closes après d'intenses sessions de lobbying de la part de l'industrie. Ce choix, en effet, déterminera l'avenir économique, social et environnemental du pays. ${ }^{11}$

Toute argumentation produisant une contre-argumentation, le raisonnement écologique réanime la figure de la défense de l'économie de l'innovation. Familier de ce type de contre-discours, Claude Allègre menace l'Europe d'un irrémédiable déclin si elle ne saisit pas le contexte de crise économique pour relancer l'innovation technologique, usant d'une énumération de dossiers hétérogènes comme arsenal rhétorique ${ }^{12}$ :

La solution, la seule, l'unique, c'est l'innovation technologique, avec comme condition première l'énergie à bon marché. Mais on ne provoquera pas la croissance si on est hostile au progrès scientifique, au nucléaire, aux OGM, aux nanotechnologies, aux cellules souches... et aux gaz et huile de schiste! Une telle attitude, hostile ou méfiante vis-à-vis du progrès, tourne le dos à la croissance. Elle génère un sentiment de peur et entraîne un climat de soupson qui envahit désormais l'Europe et annonce, hélas, son irrémédiable déclin. ${ }^{13}$

On peut lire cette séquence comme l'activation d'une prophétie de malheur. Mais elle s'organise avant tout comme l'annonce d'un point de bifurcation (tipping point) imminent, supposé donner une portée argumentative à la critique de la critique. On trouve néanmoins des figures plus nuancées, comme chez Pierre Gadonneix. Ancien PDG d'eDF, toujours très actif dans les milieux de l'énergie, notamment au sein du World Energy Council, il considère que les choix technologiques de demain doivent impérativement être faits dès aujourd'hui :

Si, dans un avenir proche, la sûreté des installations énergétiques fait enfin l'objet d'une concertation commune à tous les énergéticiens au niveau international - concertation à laquelle j’appelle vigoureusement - l'extraction des gaz non conventionnels ne devrait pas y échapper. Au-delà, cette hausse des coûts de l'énergie rend dès aujourd'bui nécessaire la mise en place de politiques publiques de l'énergie à long terme planifiant dès maintenant les investissements dont nous aurons besoin à l'avenir, quand le gaz de schiste entre autres coûtera de nouveau plus cher... Bien entendu, cette hausse des coûts rendra nécessaire des dispositifs particuliers pour ne pas pénaliser les plus pauvres et leur garantir un accès à l'énergie. Concrètement, c'est dire qu'il faut dès maintenant déployer massivement les technologies propres déjà

\footnotetext{
${ }^{11}$ Greenpeace Canada, «L'avenir éco-énergétique du Québec ne passe pas par le gaz de schiste », communiqué de presse, 11 novembre 2010.

${ }_{12} \mathrm{Au}$ sens de Marc Angenot, «Les arsenaux argumentatifs », séminaire De l'alerte au conflit, EHESS, 6 mai 2011.

${ }^{13}$ Claude Allègre, « Monsieur Hollande, n’ayez pas peur des gaz de schiste ! », Le Point, 25 août 2012.
} 
matures ; c'est dire et répéter qu'il faut désormais soutenir massivement les technologies ayant un fort potentiel et les porter à maturité. ${ }^{14}$

Les industriels ne sont pas les seuls défenseurs des gaz de schiste. A l'Assemblée nationale, les partisans de cette nouvelle ressource énergétique soutiennent qu'en stoppant l'exploration on en interdirait définitivement l'exploitation, ce qui reviendrait à créer une situation irréversible, dont une des conséquences serait un retard économique supplémentaire :

Or, l'avenir se préserve à plusieurs niveaux : il faut se préoccuper de l'environnement bien sûr, mais également de l'approvisionnement en énergie du territoire national et de la concurrence énergétique, qui est sans doute le défi majeur que nous aurons à relever dans les vingt-cinq prochaines années. Arrêter complètement quelque chose, c'est rendre extrêmement difficile d'y revenir par la suite, parce que l'opinion publique est alertée. ${ }^{15}$

En France, les instances officielles ont bien du mal à sortir de la problématique de l'« acceptabilité sociale » qui a pourtant montré ses limites sur de multiples dossiers - comme les OGM ou les nanotechnologies. La crainte d'une perte de maîtrise du processus, qui serait occasionnée par de véritables débats contradictoires dans lesquels des acteurs aux intérêts divergents explorent une pluralité de voies possibles, se traduit par un recours systématique à l'argument de la nécessité incarné ici par la contrainte d'approvisionnement énergétique.

\section{La scénarisation des futurs énergétiques sous contrainte climatique}

La controverse sur les gaz de schiste (se) nourrit de réflexions plus larges concernant l'avenir socio-économique au niveau planétaire. Qu'il s'agisse des conséquences de l'épuisement du pétrole ou du réchauffement climatique d'origine anthropique, les acteurs entreprennent de réviser les modèles du futur énergétique, en tentant de résoudre l'équation qui met en balance une consommation d'énergie fondée sur des ressources épuisables et une production génératrices de problèmes environnementaux.

Au moins depuis le rapport Stern (2006), aucun raisonnement économique à l'échelle globale ne peut être sérieusement argumenté sans intégrer les changements climatiques et environnementaux. Les scénarios développés par le GIEC servent d'appuis pour l'élaboration et la discussion des espaces de calcul ${ }^{16}$, créant une forme d'économie cognitive - par exemple à travers l'utilisation des volumes d'émissions de $\mathrm{CO}_{2}$ comme variable universelle - et ceci en dépit de l'absence d'accord politique international et les incertitudes qui pèsent sur les suites du protocole de Kyoto, après Copenhague (2009) ${ }^{17}$ puis Doha (2012). L'argument du nucléaire comme énergie à faible émission de gaz à effet de serre a été utilisé pendant plus d'une décennie

\footnotetext{
14 Pierre Gadonneix, « Gaz non conventionnels : miracle ou mirage », Le Monde, 16 septembre 2010.

15 Compte rendu de l'Examen de la proposition de loi visant à abroger les permis exclusifs de recherches d'hydrocarbures non conventionnels et à interdire leur exploration et leur exploitation sur le territoire national (n³301), Assemblée Nationale, 4 mai 2011, séance de 9h30, compte rendu n45.

16 Voir Amy Dahan-Dalmedico (dir.), Les modèles du futur. Changement climatique et scénarios économiques : enjeux politiques et économiques, Paris, La Découverte, 2007.

17 A.Dahan, S.Aykut, C.Buffet, A.Viard-Crétat: «Les Leçons politiques de Copenhague : Faut-il repenser le régime climatique?", Rapport de Recherche, Koyré Climate Series, n², Février 2010, http://www.koyre.cnrs.fr/IMG/doc/KOYRE_CLIMATE_2.doc.
} 
par les acteurs de la filière atomique. Provoquant une rupture dans les scénarisations du futur, l'accident de Fukushima a de fait servi la cause des opposants à l'atome civil, qui n'avaient jusqu'alors dans leur arsenal argumentatif que la montée des événements extrêmes (tempêtes, sécheresses, incendies, inondations) pour contrer l'idée d'un nucléaire «sauveur de la planète ${ }^{18}$. Dans le cas des gaz de schiste, le bilan carbone est rapidement jugé problématique, de sorte que leur exploitation entre plus difficilement dans l'équation énergie-climat ${ }^{19}$. Une fois de plus les États-Unis apparaissent comme les mauvais élèves de la cause climatique.

L'extraction de gaz de la roche-mère a en effet été lancée massivement aux États-Unis face à la perspective d'un épuisement des réserves de pétrole, du moins de la part des gisements jusqu'alors peu coûteux à exploiter. Qu'il s'agisse d'une diminution directe de la production de pétrole ou d'une conséquence de l'augmentation des coûts de sa mise à disposition, les gaz de schiste sont appelés à en remplacer une fraction croissante dans un nouveau « mix énergétique ». Les raisonnements s'appuient sur l'évaluation de stocks des différentes ressources dont la plupart seront épuisés à terme, avec des possibilités de renouvellement inaccessibles à l'échelle humaine.

Les débats ont depuis longtemps mis en scène un "pic pétrolier» ou "peak oil», qui correspond à l'origine à l'apex (le sommet) de la courbe de production de pétrole avant sa baisse irrémédiable, appelée «déplétion», du fait de l'épuisement des réserves de pétrole exploitables. Selon les théories de Marion King Hubbert dans les années $1940-1950^{20}$, ce pic de production était envisagé, pour les USA, autour de l'année 1970. Une des difficultés de l'exercice était d'estimer correctement les découvertes de gisements encore à venir et l'amélioration des capacités d'extraction. Repris par le Club de Rome dans The limits to growth en 1972, l'argument du pic devient le pilier de la remise en cause des vertus universelles et univoques d'une économie politique fondée sur la croissance. Dans un univers de ressources finies, il faut désormais anticiper les pénuries et contrer les transformations environnementales provoquées par la croissance - problématique dont on est loin d'être sorti, en dépit des incantations des multiples sommets internationaux ${ }^{21}$. En agitant la perspective d'un effondrement, les « peakistes » et autres «survivalistes» n'ont pas toujours une vision absolument pessimiste et fermée du devenir collectif : reconnaitre que l'humanité est face à un processus inquiétant n'interdit pas d'essayer de le retourner avant la crise.

Les calculs et les estimations de Hubbert ont engendré de multiples contestations ${ }^{22}$, et au fil du temps, la notion a évolué en donnant lieu à toute une série de réinterprétations. C'est le cas, par exemple, lorsqu'on ne raisonne plus en termes de ressources disponibles, mais plutôt en termes

\footnotetext{
${ }^{18} \mathrm{~F}$. Chateauraynaud, «Sociologie argumentative et dynamique des controverses : l'exemple de l'argument climatique dans la relance de l'énergie nucléaire en Europe ", A contrario, 2011/2 (n¹6); Benjamin Dessus et Bernard Laponche, En finir avec le nucléaire. Pourquoi, comment, Paris, Seuil, 2010.

${ }^{19}$ P. Criqui et alii., «Les scénarios énergie-climat. Mise au point après la crise, Fukushima, Durban..., et les gaz de schiste », Futuribles, n³90, novembre 2012, 5-23.

${ }^{20}$ M. King Hubbert, "Nuclear Energy and the Fossil Fuels 'Drilling and Production Practice'», in American Petroleum Institute, Drilling and Production Practice, 1956.

${ }^{21}$ Le texte final de l'ONU produit lors du dernier Sommet de la Terre $($ Rio +20$)$ dit par exemple : « We recognize that urgent action on unsustainable patterns of production and consumption where they occur remains fundamental in addressing environmental sustainability, and promoting conservation and sustainable use of biodiversity and ecosystems, regeneration of natural resources, and the promotion of sustained, inclusive and equitable global growth. », United Nations, The Future We Want, 19 juin 2012.

22 Par exemple par Steven M. Gorelick, Oil Panic and the Global Crisis: Predictions and Myths, Wiley-Blackwell, 2009.
} 
d'interactions économiques entre production et consommation qui, tout en provoquant une augmentation du prix du pétrole, peuvent créer une pénurie anticipée. La figure du pic atteint avant l'effondrement apparait comme une version simplifiée qui rend la théorie plus performative en augmentant sa puissance d'expression. Mais pour résister aux épreuves de réalité, le modèle doit tenir compte de l'évolution des prix, avec ses plateaux, ses effets d'échappement et ses micro-variations. Le raisonnement par les conséquences prend ici toute son importance puisqu'il s'agit d'introduire de plus en plus d'éléments du futur dans les scénarios, notamment ceux qui portent à envisager l'abandon progressif du pétrole et ses multiples usages, surtout dans l'industrie chimique.

Mais comment recouper des données issues d'estimations souvent sujettes à une large marge d'erreur? Pour les spécialistes et les modélisateurs, il faut connaitre avec un minimum de précision l'état des « réserves », en évaluer correctement les possibilités d'extraction, et mettre au point un modèle interprétatif de l'évolution des prix sur le marché mondial, avec ses jeux d'acteurs, ses décisions politiques et ses enjeux géostratégiques. Chacune de ces informations reste éminemment instable: les réserves évoluent avec les améliorations techniques qui permettent la rentabilité de sources ou de gisements jugés jusqu'alors négligeables, les variations mêmes du prix du pétrole définissent le curseur au-dessus duquel l'extraction est rentable, nouvelle production qui peut à son tour bouleverser le cours du baril. En matière financière, les marchés de niches ont tendance à développer facilement, dès leur naissance, des bulles spéculatives, et la ronde des énergies dites «d'avenir », qui profitent souvent du soutien de l'État, secoue les prévisions entre montées fulgurantes et dégonflement plus ou moins brutal, des promesses associées aux technologies solaires, éoliennes, ou aux gaz de schiste. Il faut donc tenir compte dans les calculs d'éventuels renversements des facteurs de l'équation, mais aussi des jeux de prévisions haussières et baissières qu'anticipent les acteurs du marché des matières premières ${ }^{23}$.

Les gaz non conventionnels (GNC), qui sont, pour les uns, une roue de secours provisoire du système et, pour les autres, un élément essentiel du futur "âge d'or du gaz », peuvent être présentés avec la même équation :

Pour autant, rappellent-ils [les experts du JRC (Joint Research Centre) et de la Direction générale de l'énergie de la Commission Européenne], la connaissance seule des réserves ne suffit pas à prédire l'avenir européen des GNC. En plus de gisements très riches, la révolution énergétique en cours aux États-Unis a aussi été rendue possible grâce à l'important maillage de gazoducs. ${ }^{24}$

On observe toujours une compétition entre le juste dosage entre production et consommation d'un côté et l'évolution des prix de l'autre pour former le bon espace de calcul permettant des prévisions suffisamment robustes :

Surtout, le gaz de schiste représente une solution au problème du "pic» pétrolier et gazier - le moment où la production commence à décliner - qui se profile en Algérie. Si l'on en croit la «BP Statistical Review », une référence dans les milieux énergétiques, le pays dispose de dix-buit années de réserves pétrolières au taux actuel de production et de cinquante années en ce qui concerne le gaz:

\footnotetext{
${ }^{23}$ On peut encore évoquer la manipulation des prix par le jeu d'estimations officielles trompeuses utilisées comme arme géopolitique.

${ }^{24}$ Journal de l'Environnement, «Bruxelles dépose le bilan des gaz de schiste », 10 septembre 2012.
} 
En fait, nombre d'experts s'attendent à voir intervenir le pic bien avant : pour le pétrole, dès 2020, pour le gaz, vers 2030. Il est peu probable que l'on découvre de fortes réserves d'bydrocarbures conventionnels. Bien qu'on ait foré "nettement plus» dans le pays durant la décennie 2000 que durant la précédente, observe le consultant Mohamed Said Beghoul dans le quotidien El Watan du 25 mars, "on a découvert nettement moins d'bydrocarbures». Un autre facteur inquiète les analystes: l'augmentation rapide de la consommation intérieure de gaz, qui réduit d'autant les possibilités d'exportation. Le prix $\mathrm{du}^{3}$ de gaz n'est que de 0,20 euro, comme celui du litre d'essence. "Le prix de l'énergie est trop bas et conduit au gaspillage, dit Tewfik. Hasni, un ancien dirigeant de la Sonatrach. La consommation nationale de gaz a quasiment doublé en dix ans. On ne pourra jamais satisfaire les objectifs d'exportation si cette tendance se poursuit. . ${ }^{25}$

L'écueil formé par l'usage non durable de ressources finies produit potentiellement des effets identiques pour d'autres ressources et matières premières, comme les métaux et les terres rares, autres dossiers qui ne cessent de s'affirmer dans le jeu des scénarios économiques. De fait, il n’y a pas un pic mais des pics, chacun pouvant être un facteur bloquant ou limitant pour une industrie. Et chacun de ces pics n'est handicapant pour une économie basée sur la croissance que dans la mesure où l'élément ne peut être remplacé, par un équivalent, par un changement technique ou par un abandon du service qui en conditionne le besoin. Le renversement du projet de société basé sur la durabilité des usages et/ou la modération de la croissance (voire de la décroissance) permet d'envisager un modèle dans lequel chacun de ces pics pourrait être levé, jusqu'à l'obtention d'un équilibre. Le pic n'est plus alors qu'une mise en scène réductrice destinée à énoncer l'inéluctable, afin, paradoxalement, de permettre l'action.

Posant l'impossibilité d'inverser la tendance, par manque de volonté politique ou par fatalité mécanique, et par conséquent l'avènement prochain d'une crise socio-économique majeure, certains acteurs s'organisent dès à présent pour se préparer au monde à venir et surtout à la période de transition. Ces preppers mettent en place des communautés de ressources, via le web, sur des forums ou des réseaux sociaux. Si tous s'accordent sur la nécessité de se préparer à la traversée d'un chaos générateur de nouvelles formes de violence ${ }^{26}$, il est plus difficile de construire un scénario solide pour l'après-pétrole. Certains auteurs-acteurs jugent très probable l'avènement d'une ère post-industrielle, relativement durable, et les plus pessimistes estiment que les excès de consommation de ressource vont annihiler tout espoir d'une nouvelle civilisation sur les ruines de l'ancienne et provoquer l'avènement d'un « nouvel âge de pierre ».

\section{La place des gaz de schiste dans le débat sur l’avenir énergétique}

Le «grand débat national sur la transition énergétique », organisé dans le prolongement de la conférence environnementale qui s'est tenu les 14 et 15 septembre 2012, a pour but de préparer une loi de programmation prévue pour fin $2013^{27}$. Au vu de l'ampleur des choix énergétiques en jeu, ce programme politique prend, aux yeux des ONG (Greenpeace, les Amis de la Terre) qui

\footnotetext{
${ }^{25}$ Hervé Kempf, «L’Algérie hésite entre le gaz de schiste et le soleil », Le Monde, 17 mai 2012

${ }^{26}$ Sur le modèle de la critique d'anticipation développée par Harald Welzer dans Les Guerres du climat. Pourquoi on tue au XXI siècle, Paris, Gallimard, 2009.

${ }^{27} \mathrm{La}$ forme d'urgence imposée par l'agenda gouvernemental évoque les tensions propres à l'accélération sociale selon Hartmut Rosa, Accélération. Une critique sociale du temps, Paris, La Découverte, 2010.
} 
ont quitté le débat avant même son lancement fin novembre 2012, l'allure d'un véritable passage en force, même si la pérennité du dispositif doit être assurée par une conférence environnementale annuelle. L'idée qui prédomine au niveau politique est celle d'un encadrement par l'État d'une réforme en profondeur de la production et de la consommation énergétique. Pour les plus radicaux, cela passe par un service public de l'énergie.

L'avenir nous commande, non pas de détruire (ou pomper) le potentiel de la nature et de ses ressources, mais d'inscrire nos activités dans les paysages en accord avec les possibilités naturelles de chaque région. Le tissu socio-économique est plus riche sans gaz de schiste qu'avec. Enfin, ces décisions gouvernementales, loin des besoins sociaux locaux, montrent combien il est nécessaire de se tourner vers la sobriété énergétique et le développement des énergies renouvelables propres. Nous n'imposerons cette politique que par une nationalisation des grands groupes énergétiques, permettant de refonder un service public de l'énergie, décentralisé, géré démocratiquement par les salariés et les usagers. ${ }^{28}$

Pour les écologistes, il s'agit d'actionner enfin le moteur du changement vers un couple production/consommation durable :

Serons-nous, cette fois, assę forts pour imposer une réelle discussion? Sans une nouvelle définition de nos besoins, qui passe par de fortes contraintes imposées à tous les process industriels, suivie d'une diminution drastique de la consommation par habitant, et d'un développement sans précédent des énergies renouvelables, il n'y a aucun avenir possible. Et au fond, tout un chacun le sait bien. ${ }^{29}$

Dans le camp opposé, l'argument économique est brandi par ceux qui estiment que le gisement potentiel de la France lui permettra d'emboîter le pas à la «nouvelle révolution industrielle», jugée déjà à l'œuvre aux États-Unis :

Le gisement français est estimé à 5100 milliards de mètres cubes, l'équivalent de soixante-cinq à quatre-vingt-dix ans de consommation nationale. Sur le papier, l'Hexagone dispose du plus fort potentiel sur le Vieux Continent. De là à imaginer la transposition en France de l'essor industriel qui transfigure l'économie des États-Unis, seul pays au monde à exploiter le gaz de schiste à grande échelle... "On assiste à une révolution industrielle aux États-Unis, s'enthousiasme Pierre-André de Chalendar, le PDG de Saint-Gobain. Les Américains ont atteint une compétitivité fantastique grâce au gaz de schiste ». . $^{30}$

Dans cette argumentation, les GNC seraient la condition pour passer d'un âge du pétrole à un âge du gaz, permettant de conserver les équilibres dans le couple production/consommation. Fin mai 2012, le chef économiste de l'Agence internationale de l'énergie (AIE), Fatih Birol, présentait, à Bruxelles, un rapport dont l'intitulé Golden rules for a golden age of shale gas (Règles d'or pour un âge d'or des gaz de schiste) est assez explicite. On lit dans l'introduction : « Le gaz naturel est sur le point d'entrer dans un âge d'or, mais ce futur dépend de manière critique de la réussite du

\footnotetext{
28 «Tout est à nous!», No gazaran Hebdo, 3 mars 2011.

${ }^{29}$ F. Nicolino, «Des questions sur les gaz de schistes », blog Planète sans visa, 20 février 2011, http://fabricenicolino.com/index.php/?p=1068.

30 « Compétitivité : Le gaz de schiste, c'est bon pour l’industrie », L'usine nouvelle, 13 septembre 2012.
} 
développement des grandes réserves mondiales de gaz non conventionnels $»^{31}$. Pour autant le rapport souligne l'importance des impacts environnementaux de l'exploitation du gaz de schiste : «Ce n'est pas un problème, c'est LE problème, indique au Monde Fatih Birol, chef économiste de l'agence, pour qui les préoccupations environnementales ne sont pas un lobbying vert, mais un souci parfaitement légitime $»^{32}$.

La question se pose d'autant plus qu'elle croise une actualité liée à des événements marquants, qui fragilisent les scénarios, et dont l'avènement est difficile à mettre en équation. Ce fut déjà le cas avec l'accident de la plateforme Deep Water Horizon en 2010 ; et surtout avec Fukushima, qui a eu pour conséquence un ralentissement des politiques nucléaires dans le monde. Ce type d'événement peut toujours donner lieu à des procédés de relativisation, mais entraîne une modification en cascade des éléments de calcul :

Fukushima "peut modifier l'équilibre énergétique actuel», estime Fatib Birol, économiste-en-chef de l'AIE, dans un entretien au Monde le 2 avril. Selon lui, "la moindre diversité du portefeuille énergétique mondial va conduire à une hausse du coût de l'énergie, donc des prix de l'électricité. Et va peser lourdement sur nos efforts pour lutter contre le changement climatique ». De fait, si les grands pays industriels décidaient de geler leurs investissements dans le nucléaire civil, voire d'en sortir complètement, le recours - inévitable à court et moyen termes - à davantage d'énergies fossiles (pétrole, gaz, charbon...) entraînerait une forte hausse des émissions de $\mathrm{CO}_{2}{ }^{33}$

La bataille autour du nucléaire et de son avenir était au cœur de la conférence environnementale. Certains acteurs politiques avaient d'ailleurs tenté d'infléchir en amont le sens du débat, comme au cours de l'été 2012, lors de la tempête provoquée par la déclaration d'Arnaud Montebourg, pour qui le nucléaire demeure «une filière d'avenir » ${ }^{34}$. Pour une partie de la presse économique, les gaz de schiste sont le levier d'une nouvelle quête d'indépendance énergétique française, en remplacement du nucléaire ${ }^{35}$. Inutile à ce propos d'en appeler au syndrome français, car le même débat existe en dehors de l'Hexagone. Au Royaume-Uni, par exemple :

C'est demain 22 mai, qu'Ed Davey, le ministre libéral démocrate de l'énergie, doit publier l'«Energy Bill », la feuille de route énergétique du gouvernement qui promet de réduire les factures d'énergie des citoyens anglais et de sécuriser l'approvisionnement énergétique du pays. Contre toute attente, il devrait annoncer que la Grande Bretagne ne va pas se lancer dans la course au gaz. de schiste, malgré l'insistance de son collègue le ministre du budget qui considère cette énergie peu couteuse, moins émettrice de $\mathrm{CO}_{2}$ que le charbon, et qui pourrait être l'une des sources principales d'électricité au cours des prochaines années. Le gouvernement britannique semble vouloir promouvoir les énergies renouvelables et avoir entendu l'argument de l'association britannique des industriels $d u$

\footnotetext{
${ }^{31}$ IEA, Golden rules for a golden age of shale gas. World Energy Outlook Special Report on Unconventional Gas, mai 2012.

32 «L'exploitation du gaz de schiste serait aussi nocive pour le climat que le charbon », Le Monde, 30 mai 2012.

33 «Le gaz de schiste, entre eldorado inespéré et désastre annoncé », Le Monde, 23 avril 2011.

34 « Pas d'abandon du nucléaire et du gaz de schiste, selon Arnaud Montebourg », Le Monde, 17 septembre 2012.

35 «Ce que le gaz de schiste a rapporté à l'économie américaine», Challenges, 13 septembre 2012. Notons que l'expression quelque peu datée d'«indépendance énergétique » tend à céder la place dans les énoncés à celles de « sécurité énergétique » ou plus précisément de « sécurité de l'approvisionnement énergétique ».
} 
secteur: "Les réformes doivent favoriser le développement des énergies renouvelables et faire en sorte qu'elles soient au ccur de notre stratégie énergétique », conclut l'une de ses membres. ${ }^{36}$

Ou aux États-Unis :

Comme aux États-Unis, les années 1990-2000 avaient été en Europe celles d'un développement massif du gaz dans la production d'électricité, freiné depuis environ dix ans par des prix du gaz. de plus en plus élevés. Le retour de prix bas met à mal la compétitivité des autres moyens à base de charbon, de nucléaire ou d'énergies renouvelables, même en intégrant le $\mathrm{CO}_{2}$ émis par la combustion du gaz. Les durées de vie de ces divers équipements ne sont pas identiques et la vision de long terme que doit avoir notre pays en matière d'investissements de production électrique doit être révisée en prenant ces divers éléments en compte. ${ }^{37}$

Si la bataille autour des scénarios est si intense, c'est parce que les enjeux sont considérables. Dans l'espace de calcul partagé par les acteurs, toute l'économie est conditionnée par le coût de l'énergie, ce qui entraîne toute une liste de conséquences socio-politiques :

Finalement, ce ne sont plus les externalités environnementales qui priment dans une analyse objective, mais globale, du projet lancé par le gouvernement (ou par l'industrie)? Pour que l'exploitation soit rentable, il faudra que le fonctionnement de l'économie tout entière s'adapte à des coûts énergétiques beaucoup plus élevés que dans le passé - probablement de $40 \%$ comme l'ont évalué les promoteurs devant le BAPE, et ce pourcentage est peut-être prudent. Nous avons déjà oublié le baril de pétrole à $147 \$$ d'il y a pas si longtemps, et nous ne planifions pas en fonction d'un baril à $200 \$$ à venir. [...] Cependant, les recherches des vingt dernières années sur la façon dont les facteurs naturels, y compris la rareté des ressources et diverses formes de stress environnemental, influent sur les comportements politiques et sociaux montrent que ces facteurs agissent rarement de manière isolée. Au contraire, ils interagissent de façon synergétique avec d'autres variables écologiques, institutionnelles, économiques et politiques pour entraîner une grande panoplie d'effets, dont certains se rapportent directement aux intérêts de l'État, alors que d'autres non. [...] En contribuant à affaiblir les économies locales, à accroître le chômage, à disloquer la vie des personnes dans les pays pauvres et vulnérables, le changement climatique contribuera à accroître les frustrations et la colère de centaines de millions de personnes. ${ }^{38}$

Jusqu'ici cependant les discussions ont essentiellement porté sur la possibilité ou non d'éviter les conséquences environnementales de la fracturation hydraulique et sur la part des gaz de schiste dans le remplacement progressif du pétrole. La pertinence de leur exploitation dans des régions aussi densément peuplées que la France reste au cœur des débats. Si certains arguments font valoir une possible rentabilité financière à moyen terme de l'extraction des GNC pour les industriels, compensée cependant par la baisse importante des prix du gaz qu'elle peut entraîner, la question de la rentabilité énergétique est régulièrement posée. En effet, la somme de l'énergie

\footnotetext{
36 « Gaz de schiste made in UK: this is the end », Journal de l'Environnement, 21 mai 2012.

${ }^{37}$ Etienne Beeker, Les gaz non conventionnels : une révolution énergétique nord-américaine non sans conséquences pour l'Europe, note d'analyse 215, Centre d'analyse stratégique, mars 2011.

38 Harvey L. Mead, «L'exploitation des gaz de schiste - Quelques enjeux économiques », Économie autrement, 20 octobre 2010, http:/ /www.economieautrement.org/spip.php?article134.
} 
dépensée pour extraire, traiter et déplacer proprement et utilement ces gaz - en l'absence d'infrastructures adéquates, c'est-à-dire de réseaux efficients de gazoducs -, est jugée problématique. Pour des auteurs comme Thomas Homer-Dixon ou Joseph Tainter, le rapport entre énergie utilisable et énergie dépensée est finalement un bon indicateur de l'évolution et de la chute des civilisations ${ }^{39}$.

\section{Conclusion}

Les transitions énergétiques ont historiquement porté des modifications sociétales majeures et durables. Qu'il s'agisse de basculer dans l'économie du gaz ou vers une part toujours plus grande d'énergies alternatives, la fin du monde du pétrole, qui a battu la mesure des événements géopolitiques mondiaux tout au long du $\mathrm{Xx}^{\mathrm{e}}$ siècle, engage les États dans des changements difficilement prévisibles. C'est, du même coup, un nouvel espace qui s'est ouvert pour les prévisionnistes, prospectivistes et autres prophètes. Quel que soit le scénario qui l'emporte, réordonner les priorités du mix énergétique ne peut que produire des réagencements dans les jeux de pouvoir. Face aux États et aux multinationales, bien souvent plus puissantes que les États, qui concentrent les pouvoirs financiers et décisionnels, on peut s'attendre à voir émerger des modalités locales de gestion, de production et d'échange de l'énergie: les mouvements qui s'organisent autour des alternatives énergétiques bénéficiant de longues années d'expériences et d'apprentissages dans d'autres secteurs comme l'environnement, l'agroécologie ou l'alimentation, monteront en puissance dans les prochaines années. Au niveau micro, l'enjeu est de défendre ses droits, tout en œuvrant à leur redéfinition : au droit à la sécurité, à l'information, au service public, à la représentation et à la participation, à l'éducation, à l'environnement et à la santé, vient ainsi s'ajouter le droit à l'énergie de plus en plus invoqué dans les arguments collectifs ${ }^{40}$. Mais dans le champ de forces qui se dessine, la défense des intérêts stratégiques majeurs, la consolidation des grandes infrastructures et la pérennité des institutions qui les contrôlent risquent fort de conduire à un durcissement des décisions régaliennes et à la production d'un nouvel agencement de pouvoirs liant les États et les firmes multinationales.

\footnotetext{
39 Thomas Homer-Dixon, The Upside of Down: Catastrophe, Creativity, and the Renewal of Civilization, Random House Canada, 2006 ; Joseph A Tainter, The Collapse of Complex Societies, Cambridge University Press, 1990.

${ }^{40}$ http://www.ouishare.net/2012/09/open-source-energy-project/
} 